\title{
Parâmetros antropométricos e indicadores de desempenho em atletas da seleção brasileira feminina de basquetebol
}

\author{
Anthropometric profile and indicators of playing performance \\ in Brazilian women's olympic basketball teams
}

João Antonio Nunes ${ }^{1,2}$ Marcelo Saldanha Aoki Leandro Ricardo Altimari ${ }^{4}$

Edio Luiz Petroski

Dante de Rose Júnior ${ }^{3}$

Paulo Cesar Montagner ${ }^{2}$

1 Confederação Brasileira de Basketball, Rio de Janeiro, RJ. Brasil.

2 Universidade Estadual de Campinas. Faculdade de Educação Física. Campinas, SP. Brasil.

3 Universidade de São Paulo. Escola de Artes, Ciências e Humanidades. Curso de Ciências da Atividade Física. São Paulo, SP. Brasil.

4 Universidade Estadual de Londrina. Departamento de Educação Física. Londrina, PR. Brasil.

5 Universidade Federal de Santa Catarina, Departamento de Educação Física. Florianópolis, SC. Brasil.

Recebido em 18/12/07 Aprovado em 24/07/08
Resumo - O presente estudo teve como objetivo investigar o nível de correlação entre os parâmetros antropométricos (peso, altura, percentual de gordura corporal, IMC) e os indicadores de desempenho (tempo de jogo, arremessos, rebotes, bolas recuperadas e erros) em atletas da seleção brasileira feminina de Basquetebol. O estudo foi conduzido durante os Jogos Olímpicos de 2000 e 2004. Os dados foram analisados por ANOVA com efeitos aleatórios. Também foram determinados os coeficientes de correlação simples de Pearson. Não foram detectadas diferenças no perfil antropométrico e no escore de indicadores de desempenho entre as duas seleções. Foi observada correlação significativa entre a estatura e o número de rebotes $(\mathrm{r}=0,61, \mathrm{p}<0,05)$. A massa magra também apresentou correlação com o número de rebotes executados $(r=0,45, p<0,05)$. Com relação ao IMC, foram observadas correlações com o tempo de jogo $(\mathrm{r}=-0,51, \mathrm{p}<0,05)$, o número de pontos $(\mathrm{r}=-0,50$, $\mathrm{p}<0,05)$, o número de erros $(\mathrm{r}=0,53, \mathrm{p}<0,05)$ e o número de bolas recuperadas $(\mathrm{r}$ $=-0,43)$. O percentual de gordura apresentou um padrão de correlação similar ao observado para o IMC (tempo de jogo: -0,51; número de pontos: -0,44; erros: 0,50 e bolas recuperadas: 0,51$)(\mathrm{p}<0,05)$. Através dos resultados obtidos, é possível afirmar que existe relação entre os parâmetros antropométricos e os indicadores de desempenho em atletas de Basquetebol. Os resultados do presente estudo sugerem que a estatura e a massa magra influenciaram a capacidade de realizar rebotes. Além disto, é plausível assumir que o conteúdo de gordura corporal exerceu influência sobre a maioria dos indicadores de desempenho analisados.

Palavras-chave: Composição corporal; Antropometria; Desempenho; Basquetebol; Atletas; Jogos Olímpicos; Feminino.

Abstract - The aim of the present study was to investigate the correlation between anthropometric parameters (weight, height, body fat, BMI) and performance indicators (playing time, points, rebounds, errors, steals) in female national basketball players. The study was conducted during the 2000 and 2004 Olympic Games. Data were analyzed by ANOVA with random effects. Pearson's simple correlation coefficients were also determined. No difference in anthropometric profile or performance indicators was observed between the two national teams (2000 and 2004). There was a significant correlation $(r=0.61, p<0.05)$ between height and number of rebounds. Free fat mass was also correlated with the number of rebounds performed $(r=0.45, p<0.05)$. BMI showed moderate correlations with playing time $(r=-0.51, p<0.05)$, points scored $(r=$ $-0.50, p<0.05)$, number of errors $(r=0.53, p<0.05)$, and number of steals $(r=-0.43$, $p<0.05)$. A similar pattern of correlation was observed between relative fat mass (\%) and performance indicators (playing time: -0.51 , points scored: -0.44 , errors: 0.50 , and steals: 0.51) $(p<0.05)$. The present results indicate a relationship between anthropometric profile and performance indicators. The results also suggest that height and free fat mass influence rebound performance. In addition, it is reasonable to assume that body fat interferes with most of the performance indicators analyzed.

Key words: Body composition; Anthropometry; Performance; Athletes; Basketball; Olympic Games; Female. 


\section{INTRODUÇÃO}

O lema "Citius, Altius, Fortius", usado para caracterizar os atletas olímpicos, reforça o conceito que o sucesso no esporte depende da seleção criteriosa dos indivíduos mais aptos e do desenvolvimento/aprimoramento de capacidades e habilidades específicas. Estes processos de seleção e treinamento são responsáveis pela superação dos limites da performance humana.

Neste contexto, as variáveis antropométricas podem ser utilizadas como indicadores relevantes para a seleção e a detecção de possíveis talentos esportivos. Corroborando esta hipótese, diversos estudos sugerem que as medidas antropométricas são variáveis que podem contribuir para o sucesso em uma determinada modalidade ${ }^{1-6}$. Além disto, nos esportes coletivos, o perfil antropométrico pode determinar a posição/função desempenhada pelos atletas ${ }^{7,8,9}$.

Alguns estudos prévios investigaram a relação entre as características antropométricas e os parâmetros funcionais/fisiológicos, que podem influenciar o desempenho físico e técnico de atletas de diferentes modalidades ${ }^{7-11}$. Porém, no Basquetebol, estas pesquisas ainda são escassas. Ostojic et al. ${ }^{8}$ demonstraram uma forte correlação entre a composição corporal e a potência aeróbia em atletas de Basquetebol. Por exemplo, neste estudo foi observado que armadores e alas apresentam maior $\mathrm{VO}_{2 \max }$ e menor percentual de gordura em relação aos pivôs. Ainda no Basquetebol, um outro estudo interessante investigou a relação entre uma capacidade física (força) e um gesto técnico do esporte (lance livre) ${ }^{12}$. Neste estudo, Kinnunen et al..$^{12}$ verificaram que a força de preensão palmar e o acerto de lances livres são variáveis que estão relacionadas. É importante ressaltar que o Basquetebol atual exige, em sua essência complexa, agilidade, velocidade e força. Também é notório o alto nível de contato físico que ocorre durante o jogo. Consequentemente, a força e a potência muscular são fundamentais neste esporte. Esta capacidade de produzir força/ potência parece ser otimizada pelo perfil cineantropométrico adequado ${ }^{14,15}$.

Partindo do pressuposto que o aspecto morfológico pode exercer importante influência sobre o rendimento esportivo, Ramana et al. ${ }^{10}$ ressaltam que a relação entre a composição corporal de atletas e o seu desempenho precisa ser mais investigada. Para estes autores ${ }^{10}$ o estudo sobre o perfil antropométrico de atletas tem como objetivo definir uma condição morfológica "ideal", específica de cada esporte. Isto possibilitaria a avaliação e a detecção destas características em jovens atletas, nas fases iniciais do seu desenvolvimento. Assim como outros autores ${ }^{2,5,10,13}$, Ramana et al..$^{10}$ também acreditam que a avaliação antropométrica é uma ferramenta que deve ser utilizada, tanto no processo de seleção de atletas, quanto no monitoramento dos efeitos do treinamento. É possível afirmar que os processos de adaptação induzidos pelo estímulo do treinamento (ex. aquisição de massa muscular e redução de massa gorda) influenciam o perfil antropométrico dos atletas ${ }^{6}$. Logo, a antropometria pode ser considerada uma estratégia complementar para o controle do processo de treinamento.

Considerando que o perfil antropomético, hipoteticamente, poderia influenciar o desempenho de atletas, o presente estudo teve como objetivos: a) avaliar e comparar o perfil antropométrico e o escore de indicadores de desempenho de duas seleções brasileiras de Basquetebol e b) investigar o nível de correlação entre as variáveis antropométricas e os indicadores de desempenho em atletas de Basquetebol.

\section{PROCEDIMENTOS METODOLÓGICOS}

A amostra do presente estudo foi constituída por 18 atletas que participaram dos Jogos Olímpicos de Sidney (2000) e Atenas (2004). Cada seleção foi constituída de 12 atletas, no entanto, vale ressaltar que um terço da amostra $(n=6)$ participou das duas Olimpíadas e os outros dois terços $(n=12)$ participaram de apenas uma delas. O estudo foi conduzido em momentos distintos, no período de 16 a 30 de setembro de 2000 e 14 a 28 de agosto de 2004. Anteriormente ao processo de coleta dos dados, as atletas foram esclarecidas em relação aos objetivos e procedimentos da pesquisa. As atletas concordaram em participar voluntariamente do procedimento experimental, assinando um termo de consentimento livre. $\mathrm{O}$ procedimento experimental foi aprovado pelo Comitê de ética em pesquisa da UNICAMP.

\section{Variáveis antropométricas}

As avaliações antropométricas de peso e estatura seguiram o protocolo proposto por Matsudo ${ }^{16}$. Para a mensuração do peso (massa corporal total), 
utilizou-se uma balança da marca Filizola ${ }^{\circledR}$, modelo 31, calibrada com precisão aproximada de $0,5 \mathrm{~kg}$. A estatura foi mensurada através de um estadiômetro de madeira com escala de medida $\mathrm{em} \mathrm{cm}$.

Para determinação das dobras cutâneas, foi utilizado um compasso (adipômetro) da marca Lange ${ }^{\circledR}$. As medidas de dobras cutâneas foram realizadas sempre no hemicorpo direito pelo mesmo avaliador. Foram feitas três coletas para cada variável, sendo considerada a média dos três valores. Com o intuito de padronizar as medidas, foi observada a técnica descrita por Benedetti et al. ${ }^{17}$. A densidade corporal foi determinada através do método de Jackson et al. ${ }^{18}$. O percentual de gordura foi calculado a partir da equação proposta por Siri1 ${ }^{19}$.

\section{Variáveis técnicas (Indicadores de desempenho)}

Todas as variáveis relacionadas ao desempenho no jogo (tempo de jogo, arremessos, rebotes, bolas recuperadas e erros) foram consideradas na sua totalidade, ou seja, o somatório das variáveis em todos os jogos do Brasil realizados durante as duas Olimpíadas. As mesmas foram obtidas através do site oficial e de domínio público da FIBA (Federação Internacional de Basquetebol), no seguinte endereço eletrônico: http://www.fiba.com.

\section{Análise Estatística}

O pacote computadorizado SPSS 15.0 foi utilizado para o tratamento estatístico dos dados. Após constatação da normalidade dos dados (teste de Kolmogorov-smirnov), inicialmente, foram realizadas duas análises: a) comparação entre as 6 atletas que participaram das duas Olimpíadas (teste $t$ student para amostras dependentes) e b) comparação entre as 6 atletas que participaram somente da Olimpíada de Sidney e as outras 6 que participaram apenas dos Jogos Olímpicos de Atenas (teste $t$ student para amostras independentes). Posteriormente, foi realizada ANOVA com efeitos aleatórios para identificação de eventuais diferenças entre as seleções brasileiras femininas de Basquetebol participantes dos Jogos Olímpicos de Sidney 2000 (n=12) e Atenas $2004(n=12)$. Considerando que algumas atletas integraram tanto a Seleção Olímpica de 2000 quanto a 2004, foi necessária a utilização de ANOVA com efeitos aleatórios, uma vez que este tipo de análise considera a dependência entre os mesmos indivíduos analisados em dois momentos diferentes. Também foram determinados, os coeficientes de correlação simples de Pearson entre os parâmetros antropométricos e os indicadores de desempenho. No presente estudo, foi adotada a classificação de faixas de correlação sugerida por Giannichi ${ }^{20}$. Este autor considera uma correlação fraca entre 0 e 0,59; regular entre 0,60 e 0,79; boa entre 0,80 e 0,89 e excelente entre 0,90 e $1^{20}$. O nível de significância estatística adotado foi de p<0,05.

\section{RESULTADOS}

Na Tabela 1, são apresentados os dados referentes às variáveis antropométricas das seleções participantes dos Jogos Olímpicos de Sidney (2000) (n=12) e Atenas (2004) (n=12). Não foi encontrada diferença significativa em nenhuma das variáveis analisadas $(\mathrm{p}>0,05)$.

Tabela 1. Comparação dos parâmetros antropométricos das seleções participantes dos Jogos Olímpicos de 2000 e 2004.

\begin{tabular}{lcccc}
\hline Variáveis & $\begin{array}{c}2000 \\
(\mathrm{n}=12)\end{array}$ & $\begin{array}{c}2004 \\
(\mathrm{n}=12)\end{array}$ & $\mathrm{F}$ & $\mathrm{P}$ \\
\hline Idade (anos) & $25,6 \pm 4,5$ & $26,9 \pm 4,1$ & 0,464 & 0,509 \\
Estatura (cm) & $181,8 \pm 9,9$ & $182,6 \pm 9,6$ & 0,132 & 0,723 \\
$\begin{array}{l}\text { Peso Corpo- } \\
\text { ral (kg) }\end{array}$ & $70,3 \pm 8,1$ & $77,6 \pm 12,7$ & 2,670 & 0,130 \\
IMC (kg/m²) & $21,5 \pm 1,6$ & $23,7 \pm 2,5$ & 3,964 & 0,071 \\
$\%$ Gordura & $21,1 \pm 1,7$ & $22,0 \pm 5,0$ & 0,354 & 0,563 \\
$\begin{array}{l}\text { Massa Magra } \\
\text { (kg) }\end{array}$ & $55,4 \pm 5,8$ & $60,0 \pm 7,1$ & 3,001 & 0,111 \\
$\begin{array}{l}\text { Massa Gorda } \\
\text { (kg) }\end{array}$ & $14,9 \pm 2,7$ & $17,5 \pm 6,5$ & 1,622 & 0,228 \\
\hline
\end{tabular}

Valores expressos em média \pm desvio padrão da média. IMC - índice de massa corporal; \%G - gordura relativa. F - variância obtida na ANOVA com efeitos aleatórios.

A Tabela 2 apresenta os valores referentes aos indicadores de desempenho das duas seleções. Com relação aos indicadores de desempenho analisados, também não foi evidenciada nenhuma diferença significativa entre as equipes de $2000(n=12)$ e $2004(n=12)(p>0,05)$.

Considerando que 6 atletas participaram das duas seleções, inicialmente, foi feita uma comparação entre os parâmetros antropométricos e os indicadores de desempenho das mesmas em 2000 e 2004. Não foi detectada diferença nos parâmetros avaliados $(\mathrm{p}>0,05)$. Além disto, foi realizada outra análise, comparando as atletas que participaram apenas de Sidney (2000) $(n=6)$ e as atletas que participaram somente de Atenas (2004) (n=6). Nesta segunda análise, foi 
detectada diferença apenas para massa magra $(\mathrm{p}=0,03)$. As atletas que participaram de Atenas $(n=6)$ apresentaram maior quantidade de massa magra $(12 \%)$ em relação às outras $(n=6)$ que representaram o Brasil em Sidney. Entretanto, conforme mencionado anteriormente, ao comparar o conjunto das 12 atletas que participaram de cada uma das Olimpíadas (Sidney, $\mathrm{n}=12$ vs. Atenas, $n=12$ ), não foi verificada diferença em nenhum dos parâmetros avaliados $(p>0,05)$ (Tabelas 1 e 2).

Tabela 2. Comparações dos indicadores de desempenho das seleções participantes dos Jogos Olímpicos de 2000 e 2004.

\begin{tabular}{lcccc}
\hline Variáveis & $\begin{array}{c}2000 \\
(\mathrm{n}=12)\end{array}$ & $\begin{array}{c}2004 \\
(\mathrm{n}=12)\end{array}$ & $\mathrm{F}$ & $\mathrm{P}$ \\
\hline $\begin{array}{l}\text { Número de } \\
\text { Jogos }\end{array}$ & $6,2 \pm 0,7$ & $7,1 \pm 0,4$ & 0,902 & 0,362 \\
$\begin{array}{l}\text { Tempo de } \\
\text { Jogo (min) }\end{array}$ & $137,7 \pm 28,9$ & $133,7 \pm 24,3$ & 0,011 & 0,915 \\
Pontos & $46,8 \pm 14,6$ & $52,8 \pm 13,7$ & 0,084 & 0,777 \\
Rebotes & $21,3 \pm 6,4$ & $23,8 \pm 5,3$ & 0,057 & 0,814 \\
Erros & $9,1 \pm 2,0$ & $9,3 \pm 1,5$ & 0,004 & 0,948 \\
$\begin{array}{l}\text { Bolas Re- } \\
\text { cuperadas }\end{array}$ & $3,6 \pm 1,2$ & $5,9 \pm 1,5$ & 1,385 & 0,263 \\
\hline
\end{tabular}

Valores expressos em média \pm desvio padrão da média. F - variância obtida na ANOVA com efeitos aleatórios.

A Tabela 3 apresenta os coeficientes de correlação simples de Pearson entre as variáveis antropométricas e os indicadores desempenho. Foi detectada correlação moderada entre a estatura e o número de rebotes $(r=0,61, p<0,05)$. A massa magra apresentou correlação fraca com o número de rebotes $(r=0,45, p<0,05)$. Com relação ao IMC, foram observadas correlações fracas com o tempo de jogo $(r=-0,51, p<0,05)$, o número de pontos $(\mathrm{r}=-0,50, \mathrm{p}<0,05)$, o número de erros $(r=0,53, p<0,05)$ e o número de bolas recuperadas $(r=-0,43, p<0,05)$. De uma maneira bastante semelhante ao IMC, o percentual de gordura também apresentou correlação com os indicadores de desempenho (tempo de jogo:
-0,51; número de pontos: -0,44; erros: 0,50 e bolas recuperadas: 0,51$)(\mathrm{p}<0,05)$.

\section{DISCUSSÃO}

Os profissionais do esporte (técnicos, preparadores, fisiologistas, etc.) buscam, incessantemente, aprofundar seus conhecimentos sobre as características e as exigências específicas de cada modalidade, visando, desta forma, maximizar o desempenho dos atletas. Neste cenário, existe um grande interesse a respeito da influência do perfil antropométrico sobre o desempenho esportivo. $O$ presente estudo teve como objetivo investigar a relação entre as variáveis antropométricas e a performance de atletas de basquetebol.

Através dos resultados obtidos no presente estudo, foi constatado que o perfil antropométrico das atletas não apresentou variação significativa, mantendo-se constante entre as equipes que representaram o Brasil em Sidney (2000) e Atenas (2004) (Tabela 1). No entanto, com relação à variável massa magra $(\mathrm{kg})$, apesar de não ser considerada estatisticamente diferente, a seleção brasileira apresentou maior índice em Atenas $(60,0 \pm 7,1 \mathrm{~kg})$ quando comparada à equipe que participou dos Jogos Olímpicos de Sidney $(55,4 \pm 5,8 \mathrm{~kg})$. Estes valores são mais baixos que os encontrados por Hoare ${ }^{2}$, para as posições de armadora $(59,0 \mathrm{~kg})$, lateral $(62,5$ $\mathrm{kg})$ e pivô $(70,5 \mathrm{~kg})$ e para a média do grupo $(64,7 \pm 6,7 \mathrm{~kg})$.

Com relação ao peso corporal, as médias das seleções olímpicas do Brasil foram de 70,3 \pm 8,1 e $77,6 \pm 12,7 \mathrm{~kg}$ para Sidney (2000) e Atenas (2004), respectivamente. Vale ressaltar que este valor é superior ao reportado por Gillam ${ }^{21}$ que conduziu seu estudo com atletas de nível universitário. $\mathrm{O}$ percentual de gordura observado nas duas seleções (Sidney: 21,1 \pm 1,7\% e Atenas: 22,0 \pm 5,0\%) está de acordo com os achados de Bayios et al. ${ }^{3}$ que

Tabela 3. Coeficientes de correlação simples (r) entre os indicadores de desempenho e os parâmetros antropométricos em atletas de Basquetebol.

\begin{tabular}{|c|c|c|c|c|c|}
\hline & Tempo & Pontos & Rebotes & Erros & Bolas rec \\
\hline Estatura $(\mathrm{cm})$ & 0,14 & 0,24 & $0,61^{*}$ & $-0,02$ & $-0,11$ \\
\hline Peso Corporal (kg) & $-0,24$ & $-0,15$ & 0,35 & $-0,37$ & $-0,35$ \\
\hline $\mathrm{IMC}\left(\mathrm{kg} / \mathrm{m}^{2}\right)$ & $-0,51^{*}$ & $-0,50^{*}$ & $-0,15$ & $0,53^{*}$ & $-0,43^{*}$ \\
\hline$\% G(\%)$ & $-0,51^{*}$ & $-0,44^{*}$ & $-0,02$ & $0,50 *$ & $-0,51^{*}$ \\
\hline Massa Magra $(\mathrm{kg})$ & $-0,10$ & $-0,02$ & $0,45^{*}$ & $-0,28$ & $-0,26$ \\
\hline Massa Gorda (kg) & $-0,38$ & $-0,29$ & 0,17 & $0,44^{*}$ & $-0,44^{*}$ \\
\hline
\end{tabular}

*Coeficiente de correlação significativo $(p<0,05)$. 
encontraram um valor médio de 24,3\% para jogadoras participantes da liga nacional da Grécia.

A manutenção do perfil antropométrico entre as seleções que representaram o Brasil em Sidney (2000) e Atenas (2004) (Tabela 1) pode ser explicada pelo fato da avaliação antropométrica ser utilizada como um dos critérios de corte. Este tipo de estratégia contribuiu para que o perfil de ambas as seleções tenha permanecido homogêneo. Além disto, metade das atletas ( $\mathrm{n}=6$ ) da seleção de Sidney (2000) também participou da seleção de Atenas (2004).

A Tabela 2 apresenta as comparações dos indicadores de desempenho das seleções participantes dos Jogos Olímpicos de Sidney (2000) e Atenas (2004). Não foi encontrada diferença em nenhuma das variáveis analisadas $(\mathrm{p}>0,05)$. Apesar de não ser considerado estatisticamente diferente, o escore referente aos indicadores de desempenho (número de pontos, rebotes e bolas recuperadas) foi ligeiramente maior na Olimpíada de Atenas (2000). Segundo Sampaio ${ }^{22}$, essas variáveis são consideradas determinantes para o resultado final do jogo. Entretanto, embora, não tenha sido detectada diferença significativa no escore desses indicadores, o resultado final foi melhor em Sidney (2000), onde foi conquistada a medalha de bronze. Esse fato incita a reflexão sobre a influência de inúmeras variáveis que ainda não são avaliadas de forma objetiva, mas que também são determinantes do sucesso nos esportes coletivos.

No presente estudo, também foi investigado o nível de correlação entre os parâmetros antropométricos e os indicadores de desempenho de atletas da seleção brasileira feminina de Basquetebol. A tabela 3 apresenta os coeficientes de correlação simples de Pearson. A estatura apresentou correlação significativa com o número de rebotes realizados. Também foi observada correlação entre a massa magra e o número de rebotes. É relevante considerar que o êxito neste fundamento depende do conjunto de ações específicas que, somados à estatura, à massa magra e à impulsão vertical, possibilitam ao atleta ter sucesso nesse fundamento. Os pivôs, normalmente, são os atletas que apresentam maior capacidade de execução do rebote, provavelmente, por serem mais altos. Também é importante considerar o fato de que os mesmos jogam mais próximos ao cesto.

Com relação ao IMC e ao percentual de gordura, as duas variáveis apresentaram correlações negativas com o tempo de jogo, número de pontos e bolas recuperadas. Em contrapartida, as atletas que possuíam mais massa magra e estatura elevada apresentaram vantagem na maioria dos indicadores de performance. Este resultado é esperado, já que atletas que possuem excesso de gordura corporal têm a velocidade e a potência prejudicadas durante a partida ${ }^{23}$.

No estudo de Paiva Neto e César ${ }^{23}$, também foram observados coeficientes de correlação negativa entre a porcentagem de gordura e os parâmetros de rendimento desportivo, reforçando a incompatibilidade entre o aprimoramento do desempenho e o alto índice de adiposidade. Para atletas envolvidos em esportes que exigem saltos e sprints consecutivos, como o Basquetebol, o baixo percentual de gordura e o maior percentual de massa magra podem contribuir para a otimização da performance.

Outras pesquisas também investigaram a influência dos parâmetros funcionais e morfológicos sobre o desempenho esportivo. Recentemente, Veale et al..$^{24}$ demonstraram que a estatura é à única variável antropométrica que apresenta tendência de diferença $(\mathrm{p}=0,07)$ entre atletas que haviam, ou não, sido selecionados para compor uma equipe de futebol australiano. Neste estudo, os autores sugerem que a altura parece ter influenciado a seleção dos atletas ${ }^{24}$. Já em outro estudo, também realizado com atletas de futebol australiano, Young e Prior ${ }^{13}$ verificaram que jogadores mais altos, com maior impulsão vertical e com maior peso apresentam vantagem em alguns indicadores de desempenho técnico que podem influenciar o sucesso da equipe. Neste estudo, foi constatado que os atletas mais pesados $(6 \%)$ marcavam mais pontos no jogo. Mais especificamente no Basquetebol, Kinnunen et al. ${ }^{12}$ demonstraram que atletas com maior força de preensão palmar apresentam melhor desempenho no lance livre.

Entretanto, é importante ressaltar que nem sempre é possível estabelecer relação entre as variáveis antropométricas e o desempenho. No estudo de Ugarkovic et al. ${ }^{11}$, não foi observada correlação entre o desempenho de salto e as variáveis antropométricas. Neste estudo, os autores sugerem que tamanho corporal e a composição corporal não são indicativos confiáveis para predizer o desempenho em movimentos específicos, especialmente em uma amostra relativamente homogênea. Hoare ${ }^{2}$ afirma que o perfil antropométrico e as características fisiológicas são variáveis que podem influenciar o desempenho esportivo, entretanto, o mesmo 
enfatiza que o sucesso em uma modalidade, como o Basquetebol, é multifatorial.

\section{CONCLUSÃO}

O perfil antropométrico e o escore dos indicadores de desempenho não apresentaram diferenças significativas entre as seleções avaliadas. Os resultados do presente estudo sugerem que existe uma relação entre os parâmetros antropométricos e os indicadores de desempenho em atletas de Basquetebol. Os resultados obtidos indicam que a estatura e a massa magra são parâmetros capazes de influenciar a execução do rebote. Além disto, o conteúdo de gordura corporal também é outro parâmetro que interfere na maioria dos indicadores de desempenho, reforçando a incompatibilidade entre a adiposidade e o rendimento esportivo.

\section{REFERENCIAS BIBLIOGRÁFICAS}

1. Carter JEL. Physical Structure of Olympic Athletes. Part II. Kinanthropometry of Olympic Athletes. Basel:Karger; 1984.

2. Hoare DG. Predicting success in junior elite basketball players--the contribution of anthropometic and physiological attributes. J Sci Med Sport 2000; 3(4):391-405.

3. Bayios IA, Bergeles NK, Apostolidis NG, Noutsos KS, Koskolou MD. Anthropometric, body composition and somatotype differences of Greek elite female basketball, volleyball and handball players. J Sports Med Phys Fitness 2006; 46(2):271-280.

4. Fernandes R, Barbosa T, Vilas-Boas JP. Fatores cineantropométricos determinantes em natação pura desportiva. Rev Bras Cineantropom Desempenho Hum 2005; 7(1):30-34.

5. Mazza JC, Alarcon N, Galasso C, Bermudez C, Cosolito P, Gribaudo F. Proporcionality and anthropometric fractionation of body mass in south american swimmers. Aquatic Sports Med 1991; 230-244.

6. Barr SI, McCarger LJ, Crawford SM. Practical use of body composition analysis in Sport. Sports Med 1994; 17(5):277-282.

7. Pyne DB, Gardner AS, Sheehan K, Hopkins WG. Positional differences in fitness and anthropometric characteristics in Australian football. J Sci Med Sport 2006; 9(1-2):143-150.

8. Ostojic SM, Mazic S, Dikic N. Profiling in basketball: Physical and physiological characteristics of elite players. J Strength Cond Res 2006; 20(4):740-744.

9. Cormery B, Marcil M, Bouvard M. Rule change incidence on physiological characteristics of elite basketball players: a 10-year investigation. $\mathrm{Br} \mathrm{J}$ Sports Med 2008; 42(1):25-30.

10. Ramana YV, Surya MVLK, Sudhakar Rao S, Balakrishna N. Effect of changes in body composition profile on VO2max and maximal work performance in athletes. JEPonline 2004; 7(1):34-39

11. Ugarkovic D, Matavulj D, Kukolj M, Jaric S. Standard anthropometric, body composition, and strength variables as predictors of jumping performance in elite junior athletes. J Strength Cond Res 2002; 16(2):227-230.
12. Kinnunen DA, Colon G, Espinoza D, Overby LY, Lewis DK. Anthropometric correlates of basketball free-throw shootings by young girls. Percept Mot Skills 2001; 93(1):105-108.

13. Young WB, Pryor L. Relationship between pre-season anthropometric and fitness measures and indicators of playing performance in elite junior Australian Rules football. J Sci Med Sport 2007; 10(2):110-118.

14. Mercier B, Mercier J, Granier P, Le Gallais D, Préfaut C. Maximal anaerobic power: relationship to anthropometric characteristics during growth. Int J Sports Med 1992; 13(1):21-26

15. Kanehisa H, Kuno S, Katsuta S, Fukunaga T. A 2-year follow-up study on muscle size and dynamic strength in teenage tennis players. Scand J Med Sci Sports 2006; 16(2):93-101.

16. Matsudo VK. Testes em Ciências do Esporte. São Caetano do Sul: CELAFISCS; 1984.

17. Petroski EL. Antropometria: técnicas e padronizações. Porto Alegre: Editora Palotti; 1999.

18. Jackson AS, Pollock ML, Ward A. Generalized equations for predicting body density of women. Med Sci Sports Exerc 1980; 12(3): 175-181.

19. Siri WE. Body composition from fluid space and density. In: Brozek J, Hanschel A. editors. Techniques for measuring body composition. Washington, D.C. :National Academy of Science; 1961. p. 223-224.

20. Giannichi RS. Medidas e avaliação em Educação Física. Viçosa: Imprensa Universitária; 1984.

21. Gillam GM. Basketball Bioenergetics: Physiological basis. NSCA J 1984; 6(6):44-45.

22. Sampaio, J. Los indicadores estadisticos mas determinantes em el resultado final en los partidos de basquetbol. Lecturas: Educacion Física y Deportes. Revista Digital: Buenos Aires. 1998; año 3, n.11. Disponível em: http://www.efdeportes.com/efd11/ samp2.htm [1998 oct 01 ].

23. Neto AP, César MC. Avaliação da composição corporal de atletas de basquetebol do sexo masculino participantes da liga nacional 2003. Rev Bras Cineantropom Desempenho Hum 2005; 7(1):35-44.

24. Veale J P, Pearce A J, Koehna S, Carlson J S. Performance and anthropometric characteristics of prospective elite junior Australian footballers: A case study in one junior team, J Sci Med Sport 2008; 11(2):227-230.

\section{Agradecimentos}

Gostaríamos de expressar nossos agradecimentos ao Prof. Dr. Alex Antonio Florindo pelo relevante auxílio na análise e discussão dos resultados.

\section{Endereço para correspondência}

Marcelo Saldanha Aoki

Universidade de São Paulo. Escola de Artes, Ciências e Humanidades

Av. Arlindo Bettio, 1000. Ermelino Matarazzo 03828-000 - São Paulo, SP.

E-mail: saldanha.caf@usp.br 